\title{
The classical double copy in three spacetime dimensions
}

\author{
Mariana Carrillo González, ${ }^{a}$ Brandon Melcher, ${ }^{b}$ Kenneth Ratliff, ${ }^{b}$ Scott Watson ${ }^{b}$ and \\ Chris D. White ${ }^{c}$ \\ ${ }^{a}$ Center for Particle Cosmology, Department of Physics and Astronomy, \\ University of Pennsylvania, \\ 209 S 33rd St, Philadelphia, PA 19104, U.S.A. \\ ${ }^{b}$ Department of Physics, Syracuse University, \\ Crouse Dr, Syracuse, NY 13244, U.S.A. \\ ${ }^{c}$ Centre for Research in String Theory, \\ School of Physics and Astronomy, Queen Mary University of London, \\ 327 Mile End Road, London E1 4NS, U.K. \\ E-mail: cmariana@sas.upenn.edu, bsmelche@syr.edu, krratlif@syr.edu, \\ gswatson@syr.edu, christopher.white@qmul.ac.uk
}

ABSTRACT: The double copy relates scattering amplitudes in gauge and gravity theories, and has also been extended to classical solutions. In this paper, we study solutions in three spacetime dimensions, where the double copy may be expected to be problematic due to the absence of propagating degrees of freedom for the graviton, and the lack of a Newtonian limit. In particular, we examine the double copy of a gauge theory point charge. This is a vacuum solution in gauge theory, but leads to a non-vacuum solution in gravity, which we show is consistent with previously derived constraints. Furthermore, we successfully interpret the non-trivial stress-energy tensor on the gravity side as arising from a dilaton profile, and the Newtonian description of a point charge emerges as expected in the appropriate limit. Thus, our results provide a non-trivial cross-check of the classical Kerr-Schild double copy.

KeYwords: Black Holes, Gauge-gravity correspondence, Scattering Amplitudes

ArXiv EPrint: 1904.11001 


\section{Contents}

1 Introduction 1

2 The classical double copy 4

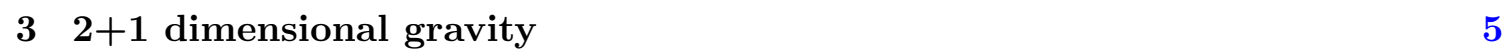

3.1 Vacuum solutions in three spacetime dimensions are flat 6

$\begin{array}{lll}3.2 & \text { Three-dimensional gravity has no Newtonian limit } & 7\end{array}$

4 The BTZ black hole $\quad 8$

4.1 Features 8

4.2 The BTZ single copy $\quad 9$

5 The double copy of a point charge $\quad 10$

$\begin{array}{lll}5.1 \text { Expecting Newtonian behavior } & 10\end{array}$

$\begin{array}{ll}5.2 \text { Point charge in } 2+1 \text { dimensions and its double copy } & 12\end{array}$

$\begin{array}{lll}5.2 .1 & \text { Newtonian-like limit } & 14\end{array}$

$\begin{array}{lll}5.2 .2 & \text { Orbits and perihelion precession } & 16\end{array}$

6 Conclusion $\quad 19$

A No fluid interpretation of the double copy stress-energy tensor 20

\section{Introduction}

The BCJ double copy, originally proposed in [1], relates scattering amplitudes in gauge and gravity theories. Specifically, it invokes color-kinematic duality, which can be defined as follows. First, one may express an $m$-point $\ell$-loop gluon amplitude as a sum over cubic graphs:

$$
\mathcal{A}_{m}^{\ell}=\sum_{g} \frac{c(g) n(g)}{d(g)}
$$

where $c(g), n(g)$, and $d(g)$ are the color factors, kinematic numerators, and propagators respectively of the cubic graph $g$, and integrals over loop momenta are left implicit. Individual terms in the sum are not unique, but mix with each other under generalized gauge transformations, consisting of conventional (local) gauge transformations and / or field redefinitions. Color-kinematics duality is then the statement that there exists a choice of generalized gauge, called color-dual gauge, in which the kinematic numerators obey the same algebra as the color factors:

$$
\begin{aligned}
c(g) & =-c(\bar{g}), & n(g) & =n(\bar{g}), \\
c\left(g_{s}\right) & =c\left(g_{t}\right)+c\left(g_{u}\right), & n\left(g_{s}\right) & =n\left(g_{t}\right)+n\left(g_{u}\right),
\end{aligned}
$$


where $\bar{g}$ denotes a graph obtained from $g$ by exchanging an odd number of vertices and the graphs $g_{t}$ and $g_{u}$ are obtained from the graph $g_{s}$ by picking an internal leg in $g_{s}$, calling it the $s$-channel, and then switching it to the $t$ - or $u$-channel, leaving the rest of the graph unchanged. The relations obeyed by the $c$ 's are automatic, following solely from the algebraic properties of the structure constants of Lie groups (i.e. the Jacobi identity). On the other hand, the fact that we can find kinematic numerators satisfying the same algebra is highly non-trivial and does not hold for any gauge invariant operator [2-4]. The BCJ double copy then states that given some such $\mathcal{A}_{m}^{\ell}$ in color-dual gauge we can obtain an $m$-point $\ell$-loop graviton amplitude by replacing the color factors with another set of kinematic numerators,

$$
\mathcal{M}_{m}^{\ell}=\sum_{g} \frac{n(g) \tilde{n}(g)}{d(g)},
$$

where the $\tilde{n}$ 's are also in color-dual gauge and need not come from the same gauge theory. The two contributing gauge theories are called the single copies of the gravity theory, which is called the double copy of the two gauge theories, where the same nomenclature is applied to the amplitudes themselves. The double copy (and / or BCJ duality) is proven at tree level [5-13], where it is known to be equivalent to the low energy limit of the socalled KLT relations in string theory [14]. There is also highly non-trivial evidence at loop level [15, 15-39], to all-orders in certain kinematic limits [40-43, 43-47], and including in the partial or complete absence of supersymmetry. In particular, the double copy of pure non-supersymmetric Yang-Mills theory is Einsteinian gravity coupled to a two-form field and a dilaton.

Both the color-kinematic duality and BCJ double copy described above are purely perturbative relations, but their seeming validity at multiple loops indicates that they may be the perturbative manifestations of some nonperturbative symmetry or duality between gauge and gravity theories, though it is as yet unknown how they may be made explicit at the Lagrangian level (see e.g. refs. [48-51] for an exploration of the latter). Motivated by this possibility, ref. [52] extended the double copy from scattering amplitudes to a certain family of exact classical solutions. Specifically, it considered stationary Kerr-Schild metrics, such that there exists a coordinate system in which its components satisfy

$$
g_{\mu \nu}=\eta_{\mu \nu}+\kappa \phi k_{\mu} k_{\nu}, \quad \partial_{0} g_{\mu \nu}=0,
$$

with $\eta_{\mu \nu}$ the Minkowski metric, $k$ a vector field which is null and geodesic with respect to $\eta$ and $g$, and $\phi$ a scalar field. Reference [52] then showed that the Einstein equations for $g_{\mu \nu}$ imply that the vector field $A_{\mu}=\phi k_{\mu}$ satisfies the abelian Yang-Mills equations of motion

$$
\partial_{\nu} F^{\mu \nu}=g J^{\mu}
$$

and is thus a well-defined single copy of the graviton $h_{\mu \nu}$. Subsequent studies extended this class of solutions to include multiple Kerr-Schild terms [53], time dependence [54], type D spacetimes [55], as well as examining how source terms are related in the two theories [56]. Furthermore, it has been shown that classical solutions can be related through the double copy even if they cannot be put in Kerr-Schild form at the expense of having 
to work order-by-order in perturbation theory [57-67]. Investigations of non-perturbative aspects have been carried out in refs. [68-71], and an alternative body work has looked at matching up gauge and gravity solutions in a wide catalogue of linearised theories, in arbitrary gauges [72-80]. In refs. [81, 82], an extension of the Kerr-Schild approach to nonMinkowski background metrics was investigated, where an interesting byproduct of ref. [81] is a general relation that must be satisfied by the source current in the gauge theory, and the stress-energy tensor in the gravity theory. For stationary solutions this relation reads: ${ }^{1}$

$$
J^{\mu}=2\left(\delta^{\mu}{ }_{0} \frac{T}{d-2}-T_{0}^{\mu}\right)
$$

where $T=T_{\mu}^{\mu}$. For completeness we note that eq. (1.6) holds only if we choose $k^{0}=+1$ and hence differs by a minus sign from the corresponding expression in [81], in which $k_{0}=+1$.

Despite the above developments, a full understanding of the double copy, including a possible underlying explanation, is still lacking. It is then interesting to consider highly unusual situations, in which the double copy might break. If it survives such non-trivial tests, our confidence in the ultimate validity of the double copy is increased, and the frontiers of its potential application extended. A suitable playground in this regard is to consider three spacetime dimensions. As we review in what follows, General Relativity in $d=3$ is straightforward to formulate, but has the unfortunate consequence that there are no propagating degrees of freedom for the graviton, and no well-behaved Newtonian limit. This is not a problem for gauge theory in three spacetime dimensions, which from the point of view of the Kerr-Schild single copy means the linearised Yang-Mills, or Maxwell, equations. In the latter case, one can easily speak of a propagating photon, and thus one might worry that degrees of freedom no longer match up in the Kerr-Schild double copy, which typically associates a gauge theory solution with a pure gravity counterpart, as above.

The ultimate resolution of this puzzle is that the double copy of gauge theory is not pure gravity but, as noted above, GR coupled to a dilaton and two-form field. This provides a possible mechanism for the Kerr-Schild double copy to work, but if and how it works must be investigated using explicit applications. We will present two examples in this paper. The first is the well-known BTZ black hole [83], first studied in the context of the Kerr-Schild single copy in ref. [81]. This is an example without a non-zero dilaton profile on the gravity side. Our second example is entirely new and consists of the double copy of a point charge in (Abelian) gauge theory. Although the point charge is a vacuum solution in the gauge theory (apart from at the origin, where the charge resides), we will see that its double copy is not a vacuum solution of the Einstein's equations. Instead, there is a non-zero stress-energy tensor throughout spacetime, which will be successfully interpreted as arising from a dilaton. Thus, the double copy relation indeed survives and provides a highly non-trivial example of the Kerr-Schild double copy: in no previous examples has the dilaton been non-zero. We will see a nice physical interpretation of its presence here,

\footnotetext{
${ }^{1}$ The non-covariant-looking presence of the components $T^{\mu}{ }_{0}$ comes from the also-non-covariant assumption that there exists a particular timelike direction along which $g_{\mu \nu}$ is constant. A covariant form which involves a general Killing vector field can be found in ref. [81].
} 
namely that it leads to geodesics that can follow stable orbits and have a well-defined Newtonian-like limit mimicking the behaviour in the gauge theory.

The structure of our paper is as follows. In section 3, we review the issues concerning gravity in $2+1$ dimensions, before examining the Kerr-Schild copy of the previously studied BTZ black hole in section 4 . In section 5 we construct the double copy of the point charge in $2+1$ dimensions, and examine its physical properties. We discuss our results and conclude in section 6 .

Conventions. We denote the gravitational coupling by $\kappa$ and define it in arbitrary dimensions by the Einstein-Hilbert action, or equivalently the Einstein equations:

$$
S_{\mathrm{EH}}=\int \mathrm{d}^{d} x \sqrt{-g}\left(\frac{1}{\kappa^{2}} R+\mathcal{L}_{\text {matter }}\right) \Longleftrightarrow G_{\mu \nu}=\frac{\kappa^{2}}{2} T_{\mu \nu} .
$$

We define Newton's constant $G_{d}$ in $d$ dimensions to be the constant of proportionality between the Newtonian potential of a unit mass and its $r$-dependence. In four spacetime dimensions these definitions implies that $\kappa$ is related to Newton's constant $G_{4}$ and the (reduced) Planck mass $M_{\mathrm{Pl}}$ by

$$
\kappa^{2}=16 \pi G_{4}=\frac{2}{M_{\mathrm{Pl}}^{2}} .
$$

However in other dimensionalities this relationship will change.

We denote a generic gauge coupling constant by $g$. When relating classical double and single copies we substitute coupling constants as

$$
\frac{\kappa}{2} \leftrightarrow g
$$

In Kerr-Schild contexts we raise and lower all indices with the Minkowski metric $\eta_{\mu \nu}$, with the exception of the indices on $R_{\mu \nu}$ and $T_{\mu \nu}$ which we raise and lower with the full metric $g_{\mu \nu}$.

We use the mostly plus metric signature $(-,+,+,+)$.

\section{The classical double copy}

The classical single copy of a stationary $d$-dimensional Kerr-Schild spacetime

$$
g_{\mu \nu}=\eta_{\mu \nu}+\kappa k_{\mu} k_{\nu} \phi
$$

can be identified as follows. The Ricci tensor with mixed indices is given by [84]

$$
\begin{aligned}
R_{\nu}^{\mu} & =\frac{\kappa}{2}\left\{\partial^{\lambda} \partial^{\mu}\left(\phi k_{\lambda} k_{\nu}\right)+\partial^{\lambda} \partial_{\nu}\left(\phi k^{\mu} k_{\lambda}\right)-\partial^{2}\left(\phi k^{\mu} k_{\nu}\right)\right\}, \\
& =\frac{\kappa^{2}}{2}\left\{T^{\mu}{ }_{\nu}-\frac{1}{d-2} \delta^{\mu}{ }_{\nu} T\right\},
\end{aligned}
$$

in the last step citing the trace-reversed Einstein equations (with $T=T_{\mu}^{\mu}$ ). Choosing $k^{0}= \pm 1$ and defining $A_{\mu}=k_{\mu} \phi, F_{\mu \nu}=\partial_{\mu} A_{\nu}-\partial_{\nu} A_{\mu}$, the $R^{\mu}{ }_{0}$ equations become

$$
\partial_{\nu}\left(\partial^{\mu}\left(\phi k^{\nu}\right)-\partial^{\nu}\left(\phi k^{\mu}\right)\right)= \pm \kappa\left\{\frac{1}{d-2} \delta_{0}^{\mu} T-T_{0}^{\mu}\right\} .
$$


Hence if we identify $A_{\mu}=k_{\mu} \phi$ and

$$
J^{\mu}= \pm 2\left\{\frac{1}{d-2} \delta_{0}^{\mu} T-T_{0}^{\mu}\right\}
$$

then eq. (2.3) reduces to

$$
\partial_{\nu} F^{\mu \nu}=g J^{\mu},
$$

which we recognize as Maxwell's equations.

To see the significance of the sign choice consider a Kerr-Schild spacetime sourced by a stationary pressureless fluid in four dimensions with a small energy density $\rho$, in which case the stress-energy tensor is $T_{\mu \nu}=\rho U_{\mu} U_{\nu}$ with $U^{\mu} \partial_{\mu}=\left(-g_{00}\right)^{-1 / 2} \partial_{0}$. Then with mixed indices we have $T^{0}{ }_{0}=-\rho, T^{i}{ }_{0}=T^{i}{ }_{j}=0$, and hence

$$
J^{0}= \pm \rho, \quad J^{i}=0 .
$$

So if we choose $k^{0}=+1$ then the double copy maps positive charge densities to positive masses and if we choose $k^{0}=-1$ then the double copy maps negative charge densities to positive masses. ${ }^{2}$ We will choose $k^{0}=+1$ so that we can identify the Kerr-Schild scalar field $\phi$ as the scalar potential on the gauge side.

However, keep in mind that on the gravity side the choice of $k^{0}$ is entirely artificial - given a Kerr-Schild graviton $k_{\mu} k_{\nu} \phi$ we can always choose $k^{0}= \pm 1$ by rescaling $k^{\mu} \mapsto$ $\pm k^{\mu} / k^{0}$ and $\phi \mapsto\left(k^{0}\right)^{2} \phi$. These redefinitions leave our spacetime unchanged but alter the gauge field with which it is identified. In flat space, choosing $k^{0}= \pm 1$ is enough to get a reasonable single copy; in $d>4$ this means mapping point sources to point sources. When considering curved space, this choice can be more complicated (see [81] for a detailed analysis).

\section{$3 \quad 2+1$ dimensional gravity}

General relativity in four spacetime dimensions is governed by the familiar Einstein-Hilbert action,

$$
S_{\mathrm{EH}}=\int \mathrm{d}^{4} x \sqrt{-g}\left\{\frac{1}{\kappa^{2}}(R-2 \Lambda)+\mathcal{L}_{\text {matter }}\right\},
$$

with $g=\operatorname{det} g_{\mu \nu}, R$ the scalar curvature, $\Lambda$ the cosmological constant, and $\mathcal{L}_{\text {matter }}$ the Lagrangian for the matter fields present. Variation with respect to the metric gives the Einstein field equations

$$
G_{\mu \nu}+g_{\mu \nu} \Lambda=\frac{\kappa^{2}}{2} T_{\mu \nu}
$$

where

$$
G_{\mu \nu}=R_{\mu \nu}-\frac{1}{2} g_{\mu \nu} R
$$

is the Einstein tensor (with $R_{\mu \nu}$ the Ricci tensor) and

$$
T_{\mu \nu}=-\frac{2}{\sqrt{-g}} \frac{\delta\left(\sqrt{-g} \mathcal{L}_{\text {matter }}\right)}{\delta g^{\mu \nu}}
$$

\footnotetext{
${ }^{2}$ Strictly speaking, the single copy maps a gravity theory to a non-Abelian gauge theory, in which case the nature of the charge is more complicated. However, colour charge is removed upon taking the double copy, which explains why a positive mass always results.
} 
the stress-energy tensor. We can straightforwardly generalize this to an arbitrary number of spacetime dimensions $d$ by

$$
S_{\mathrm{EH}}=\int \mathrm{d}^{d} x \sqrt{-g}\left\{\frac{1}{\kappa^{2}}(R-2 \Lambda)+\mathcal{L}_{\text {matter }}\right\},
$$

which leaves eq. (3.2) unchanged in form.

\subsection{Vacuum solutions in three spacetime dimensions are flat}

To see why setting $d=3$ leads to a qualitatively different theory consider the following argument, courtesy of [85]. In $d$ spacetime dimensions the Riemann tensor $R^{\lambda}{ }_{\mu \sigma \nu}$ has

$$
N_{\mathrm{R}}=\frac{1}{12} d^{2}\left(d^{2}-1\right)
$$

independent components, while the Einstein tensor $G_{\mu \nu}$ has

$$
N_{\mathrm{E}}=\frac{1}{2} d(d+1) \text {. }
$$

It follows that for $d \geq 4$ we have $N_{\mathrm{R}}>N_{\mathrm{E}}$ (i.e. $d=4$ gives $N_{\mathrm{R}}=20$ and $N_{\mathrm{E}}=10$ ). Every component of $G_{\mu \nu}(x)$ is specified at every spacetime point $x$ by the local matter distribution $T_{\mu \nu}(x)$ via the Einstein field equations (3.2), but with $N_{\mathrm{R}}>N_{\mathrm{E}}$ this cannot uniquely fix every component of $R^{\lambda}{ }_{\mu \sigma \nu}(x)$ : given $G_{\mu \nu}(x)$ the system of equations

$$
G_{\mu \nu}=g^{\lambda \sigma} R_{\lambda \mu \sigma \nu}-\frac{1}{2} g_{\mu \nu} g^{\lambda \sigma} g^{\alpha \beta} R_{\lambda \alpha \sigma \beta}
$$

which relates $R_{\mu \sigma \nu}^{\lambda}(x)$ to $G_{\mu \nu}(x)$ is a set of $N_{\mathrm{E}}$ equations in $N_{\mathrm{R}}$ unknowns and is hence underdetermined. It is precisely this freedom which allows for all the familiar gravitational phenomena we know and love: waves can propagate through the vacuum, two objects separated by empty space can gravitate toward each other, etc.

Let us now consider $d=3$ spacetime dimensions, where from the above results we have $N_{\mathrm{R}}=N_{\mathrm{E}}=6$. This implies that eq. (3.4) can be inverted, and invoking (3.2) we find

$$
R_{\lambda \mu \sigma \nu}=\frac{\kappa^{2}}{2}\left\{g_{\lambda \sigma} T_{\mu \nu}+g_{\mu \nu} T_{\lambda \sigma}-g_{\lambda \nu} T_{\mu \sigma}-g_{\mu \sigma} T_{\lambda \nu}+T\left(g_{\lambda \nu} g_{\mu \sigma}-g_{\lambda \sigma} g_{\mu \nu}\right)\right\} .
$$

In other words, the curvature $R_{\mu \sigma \nu}^{\lambda}$ at each point is determined entirely by $T_{\mu \nu}$ at that same point. In particular, the condition $T_{\mu \nu}(x)=0$ at a given spacetime point implies

$$
R_{\mu \sigma \nu}^{\lambda}(x)=0,
$$

such that no matter how much energy is contained in a given region of space, that energy will have no impact on the curvature of spacetime anywhere else. More generally, given a region $S$, the geodesics through any other region are completely insensitive to the energy and momentum distribution in $S$.

An alternate way to view this is that the graviton is overconstrained. As is well-known, the physical degrees of freedom of the graviton $h_{\mu \nu}$ can be obtained from the transverse and 
traceless part of the spatial components $h_{i j}$ of the metric. In $d$ spacetime dimensions these both amount to $d-1$ separate constraints, which if $d \geq 4$ cannot completely determine the $\frac{1}{2} d(d-1)$ independent components of a symmetric $(d-1)$-dimensional rank-two tensor. If $d=3$ on the other hand, we have $2(d-1)=4$ separate constraints on the $\frac{1}{2} d(d-1)=3$ independent components of $h_{i j}$, and are led to the conclusion that the graviton does not propagate in $d=3$.

\subsection{Three-dimensional gravity has no Newtonian limit}

We will here take the Newtonian limit of pure gravity in arbitrary dimensions and show that in $d=3$ the Newtonian potential decouples from any matter sources present.

We take the Newtonian limit as follows. We consider weak fields, i.e. fields for which there exists a coordinate system in which the metric is quasi-stationary, $\partial_{0} g_{\mu \nu} \approx 0$, and only weakly deviates from flat space, $g_{\mu \nu}=\eta_{\mu \nu}+h_{\mu \nu}$ with $\left|h_{\mu \nu}\right| \ll 1$. (In this section, we reabsorb the factor of $\kappa$ into $h_{\mu \nu}$.) We consider nonrelativistic trajectories, i.e. geodesics $x^{\mu}(\tau)$ for which $\left|\dot{x}^{i}(\tau)\right| \ll 1$ in this coordinate system, as well as a stationary weak source, i.e. a stress-energy tensor with $T_{0}^{0}=-\rho$ small and every other component vanishing. Given this, we neglect all terms beyond linear order in $\rho$ and $h_{\mu \nu}$.

It may then be shown that the spatial geodesic equation reduces to

$$
\frac{\mathrm{d}^{2} \boldsymbol{x}}{\mathrm{d} t^{2}}=\frac{1}{2} \vec{\nabla} h_{00} \equiv-\vec{\nabla} \Phi
$$

where we identify

$$
\Phi \equiv-\frac{1}{2} h_{00}=-\frac{1}{2}\left(1+g_{00}\right)
$$

as the Newtonian potential, and that the 00-component of the trace-reversed Einstein equations becomes in $d$ dimensions

$$
\vec{\nabla}^{2} \Phi=\frac{\kappa^{2}}{2} \rho\left(\frac{d-3}{d-2}\right)
$$

It is noteworthy to mention that the explicit expressions for both the Newton and Poisson equations in the Newtonian limit are gauge dependent. For example, had we worked on De Donder gauge, we would have obtained that the dimension-dependent factor appears in Newton's equation instead of in Poisson's equation [86].

Specializing to a point mass $M$ at the origin we then find the Newtonian gravitational field $\mathbf{g}=\vec{\nabla} \Phi$ a distance $r$ from the origin to be given by

$$
\mathbf{g}(r)=-\hat{\mathbf{r}}\left(\frac{M}{r^{d-2}}\right) \frac{\kappa^{2}}{2}\left\{\frac{(d-3) \Gamma\left(\frac{d+1}{2}\right)}{(d-1)(d-2) \pi^{(d-1) / 2}}\right\} .
$$

From this last equation we can then read off the $d$-dimensional Newton constant $G_{d}$ :

$$
G_{d}=\frac{\kappa^{2}}{2}\left\{\frac{(d-3) \Gamma\left(\frac{d+1}{2}\right)}{(d-1)(d-2) \pi^{(d-1) / 2}}\right\} .
$$


In $d=4$ this yields (via $\Gamma(5 / 2)=3 \sqrt{\pi} / 4)$ the expected result $G_{4}=\frac{\kappa^{2}}{16 \pi}$. However in $d=3$ we obtain $G_{3}=0$. Hence, in three spacetime dimensions the Newtonian gravitational field $\mathrm{g}$ in the presence of a point mass (or any other matter distribution, as seen from eq. (3.9)) vanishes identically at any distance. The Newtonian potential $\Phi$ is no longer coupled via Poisson's equation (3.9) to the energy distribution $\rho$, and nonrelativistic trajectories (3.7) are straight lines.

In this section, we have reviewed salient properties of three dimensional gravity, in particular the absence of propagating graviton degrees of freedom. As discussed in the introduction, this creates a potential problem for the classical double copy for exact solutions, given that the latter maps gauge boson degrees of freedom to the graviton in all known cases [52-54, 68]. To investigate this further, we first review a known three-dimensional gravitational system, and its single copy [81].

\section{The BTZ black hole}

Since gravity does not propagate in three dimensions it was long thought that there were no related black hole solutions of Einstein's equations. In 1992 Bañados, Teitelboim, and Zanelli showed [83] that in fact there do exist black hole solutions, collectively referred to as the BTZ black hole, in asymptotically AdS spacetimes. Geometrically, this black hole solution can be constructed as a quotient of the covering of $\mathrm{AdS}_{3}$ by a discrete group of isometries [87].

In the following we review the BTZ black hole in the context of the Kerr-Schild double copy which was first analyzed in [81]. For clarity, we will follow the conventions on that paper which correspond to

$$
\begin{aligned}
g_{\mu \nu} & =\eta_{\mu \nu}+k_{\mu} k_{\nu} \phi, & \\
\kappa^{2} & \rightarrow g, & M \rightarrow Q .
\end{aligned}
$$

In the rest of the paper we stick to the conventions stated previously.

\subsection{Features}

Importantly for our purposes, the BTZ spacetime admits a Kerr-Schild description [88]. More specifically, one may adopt a spheroidal coordinate system, such that the BTZ solution has the form of eq. (1.4), with the following results for the Minkowski line element, Kerr-Schild vector and scalar function respectively:

$$
\begin{aligned}
\eta_{\mu \nu} \mathrm{d} x^{\mu} \mathrm{d} x^{\nu} & =-\mathrm{d} t^{2}+\frac{r^{2}}{r^{2}+a^{2}} \mathrm{~d} r^{2}+\left(r^{2}+a^{2}\right) \mathrm{d} \theta^{2}, \\
k_{\mu} \mathrm{d} x^{\mu} & =-\mathrm{d} t+\frac{r^{2}}{r^{2}+a^{2}} \mathrm{~d} r-a \mathrm{~d} \theta, \\
\phi & =1+\frac{\kappa^{2} M}{\pi}-\frac{r^{2}}{\ell^{2}} .
\end{aligned}
$$

Here $\Lambda=-1 / \ell^{2}$ is the cosmological constant, $M$ a mass-like parameter (the charge associated with the time translation symmetry of the spacetime), and $a$ an angular momentumlike parameter (the charge associated with the rotational symmetry of the spacetime). This 
is reminiscent of the Kerr solution in $d=4$. However, unlike in the latter case, the spacetime is not sourced by a rotating disc of finite radius: it cannot be, due to the fact that gravity does not propagate in three spacetime dimensions. In fact, the above metric describes different spacetimes depending on the values of $M$ and $a$. For $M>0$ and $|a|<\ell$ we have a black hole solution that shares many features with more familiar four-dimensional black holes, the most obvious being a horizon, which turns out to be located at

$$
r_{\text {horizon }}=\ell \sqrt{\frac{M}{2}\left(1+\sqrt{1-(a / \ell)^{2}}\right)} .
$$

We note also that in the limit $a, M \rightarrow 0$ we obtain (with a coordinate change)

$$
\mathrm{d} s^{2}=-\left(\frac{r}{\ell}\right)^{2} \mathrm{~d} t^{2}+\left(\frac{r}{\ell}\right)^{2} \mathrm{~d} r^{2}+r^{2} \mathrm{~d} \theta^{2}
$$

which is not the $\mathrm{AdS}_{3}$ vacuum we would expect to find in the massless limit. Rather we obtain $\mathrm{AdS}_{3}$ with $a=0$ and $M=-1$,

$$
\mathrm{d} s^{2}=-\left(1+\left(\frac{r}{\ell}\right)^{2}\right) \mathrm{d} t^{2}+\left(1+\left(\frac{r}{\ell}\right)^{2}\right)^{-1} \mathrm{~d} r^{2}+r^{2} \mathrm{~d} \theta^{2}
$$

although every other negative value of $M$ is unphysical due to a naked conical singularity [83].

\subsection{The BTZ single copy}

Since we can write the BTZ black hole in Kerr-Schild form, we can obtain its single copy, as first presented in ref. [81]. That is, we may construct a gauge field $A_{\mu}$ such that

$$
A_{\mu} \mathrm{d} x^{\mu}=\phi k_{\mu} \mathrm{d} x^{\mu}=\left(1+\frac{g Q}{\pi}+\rho r^{2}\right)\left(-\mathrm{d} t+\frac{r^{2}}{r^{2}+a^{2}} \mathrm{~d} r-a \mathrm{~d} \theta\right)
$$

where we have relabelled $\Lambda \rightarrow \rho$. Substituting this into the Maxwell equations of eq. (1.5), one finds that the source is a constant charge density filling all space:

$$
J^{\mu}=4 \rho v^{\mu}, \quad v^{\mu} \partial_{\mu}=\partial_{t},
$$

This is consistent with the single copy of four-dimensional (Anti-)de Sitter space in ref. [53], which replaces the constant energy density in the gravity theory with a constant charge density in the gauge theory. The mass parameter $M$ for the BTZ black hole disappears in the gauge theory source: it corresponds to a constant contribution to the electrostatic potential on the gauge theory side, which has no dynamical consequences. This is in stark contrast to the Schwarzschild black hole in four dimensions, in which the point mass $M$ on the gravity side is associated with a point charge in the gauge theory. This should not surprise us, given that there is no known analogue of the Schwarzschild black hole in $d=3$. On the contrary, the unusual role of the mass parameter in the single copy serves as a further illustration of how different the BTZ black hole is to the usual Schwarzschild or Kerr black holes living in four-dimensional Minkowski space. One can also notice that; 
while the BTZ metric can describe a black hole, a particle giving rise to a conical singularity, or simply $\mathrm{AdS}_{3}$; the gauge solution does not correspond to physically different solutions when varying the free parameters $Q$ and $\rho$.

In this section, we have reviewed the well-known BTZ black hole in $d=3$ spacetime dimensions [87], which has a well-defined gauge theory single copy [81], whose interpretation (in terms of the cosmological constant) is consistent with previous results in four spacetime dimensions. We should perhaps not be at all surprised that the single copy works in this case, given that the potential difficulties of three spacetime dimensions are on the gravity side of the double copy correspondence, rather than the gauge theory side. If we start with a sensible gravity solution, we then expect a sensible gauge theory counterpart. A trickier situation would be to start with a well-defined gauge theory object, and to seek its gravitational analogue. The simplest such object is a pointlike electric charge, which we will now investigate further.

\section{The double copy of a point charge}

In all of the above examples, we have started with a gravity solution and obtained its gauge theory counterpart via the single copy. In this section, we will reverse this procedure, by starting from a gauge field and finding its corresponding Kerr-Schild double copy. This allows us to directly probe the issue of how the physical degrees of freedom in the gauge theory get mapped under the double copy. Since we will be working in $(2+1)$ dimensions, there will be no graviton degrees of freedom; instead, as already discussed in section 1, we expect to turn on the dilaton degree of freedom. Furthermore, the lack of a graviton is related to the well-known absence of a well-behaved Newtonian limit for three-dimensional General Relativity [85], although there are no such issues in the gauge theory. Let us examine this issue in more detail.

\subsection{Expecting Newtonian behavior}

Suppose we have some stationary gauge field $A_{\mu}$. In order to take its Kerr-Schild double copy we need to specify a splitting

$$
A_{\mu}=\phi k_{\mu},
$$

for some scalar function $\phi$, where $k_{\mu}$ is null and geodesic. ${ }^{3}$ Choosing $k^{0}=1$ we identify $A^{0}=\phi$, and the timelike Maxwell equation becomes

$$
g J^{0}=\partial_{\mu} F^{0 \mu}=\partial_{\mu}\left(\partial^{0} A^{\mu}-\partial^{\mu} A^{0}\right)=-\partial^{2} A^{0}=-\partial^{2} \phi=-\vec{\nabla}^{2} \phi
$$

using the stationarity of $A_{\mu}$ throughout. Thus, the scalar $\phi$ satisfies Poisson's equation with source $-J^{0}$. The field $\phi$ is thus also described by the linearised equations of biadjoint scalar theory, and can then be interpreted as the so-called zeroth copy of the gauge field $A_{\mu}$ [52].

\footnotetext{
${ }^{3}$ Note that we can only take the double copy in certain gauges, since if we choose e.g. Coulomb gauge, $A_{0}=0$, then there exists no null vector $k_{\mu}$ such that $A_{\mu}=\phi k_{\mu}$. This mimics a similar behaviour in amplitudes, namely that the double copy correspondence is manifest only in certain (generalised) gauges [15]. For a recent discussion beyond this, see ref. [89].
} 
Let us now consider the Newtonian limit of the double copy of this gauge field. With $k^{0}=1$ the purely timelike component of the metric is

$$
g_{00}=-1+\kappa \phi
$$

so the Kerr-Schild field $\phi$ is related to the Newtonian potential $\Phi$ as

$$
\Phi=-\frac{1}{2}\left(1+g_{00}\right)=-\frac{\kappa}{2} \phi .
$$

Furthermore, we can relate $\Phi$ to the source on the gravity side using eqs. (1.6) and (5.1) and making the usual substitution $\frac{\kappa}{2} \leftrightarrow g:{ }^{4}$

$$
\begin{aligned}
\vec{\nabla}^{2} \Phi & =\frac{\kappa^{2}}{4} J^{0} \\
& =\frac{\kappa^{2}}{2}\left(\frac{T}{d-2}-T_{0}^{0}\right) .
\end{aligned}
$$

This is Poisson's equation for the Newtonian potential $\Phi$, and tells us that any metric obtained by double-copying a gauge solution should have a Newtonian potential sourced by the timelike component of the gauge source, modulo replacements of charges by masses and the gauge coupling constant with its gravitational counterpart. ${ }^{5}$

The above arguments make sense in $d \geq 4$, given the existence of a well-defined Newtonian limit for gravity. Even in $d=3$ we can see that in the case of the BTZ black hole our results accord exactly with what we've already found: applying Gauss' law to a constant energy distribution (i.e. a cosmological constant) in two dimensions yields a Newtonian gravitational field satisfying $|\mathbf{g}| \propto r$, which corresponds to a potential satisfying $|\Phi| \propto r^{2}$, in agreement with eq. (4.3).

In general in the $d=3$ case eq. (5.4) becomes

$$
\vec{\nabla}^{2} \Phi=\frac{\kappa^{2}}{4} J^{0}=\frac{\kappa^{2}}{2}\left(T-T^{0}{ }_{0}\right) .
$$

That three-dimensional gravity has no Newtonian limit is down to the fact that if we take the Newtonian limit of the source as in section (3.2) then $T=T^{0}{ }_{0}=-\rho$ and the right hand side identically vanishes. From the perspective of the double copy this implies that the identification of a small charge density $J^{0}$ on the gauge side with a small energy density $T_{00}$ on the gravity side, which holds in $d \geq 4$, breaks down in three dimensions. Hence it becomes natural to ask: what gravitational source does correspond to a small charge density in three dimensions?

\footnotetext{
${ }^{4}$ This is the more general form of the relation (3.9), which we obtain from eq. (5.4) by taking the Newtonian limit of the source. (The second line of eq. (5.4) can be derived without reference to the double copy, but doing it this way clarifies the relationship between the Newtonian and electrostatic potentials and their sources.)

${ }^{5}$ Note that the minus sign in eq. (5.3) is necessary for our results to be consistent. We have already claimed that the choice $k^{0}=+1$ allows us to identify positive charges with positive masses. The negative sign in $\Phi \propto-\phi$ is crucial for this identification to hold: with positive $\rho=J^{0}$ the solutions of $\vec{\nabla}^{2} \phi \propto-J^{0}$ are repulsive while those of $\vec{\nabla}^{2} \Phi \propto+J^{0}$ are attractive.
} 
As we can see from eq. (5.5), such a source must have nonzero spatial diagonal components even in this quasi-Newtonian limit in order for $T-T^{0}{ }_{0}$ to be non-vanishing. Further, since we know that a nontrivial scalar potential $\phi$ will generically be non-vanishing everywhere, we expect that the double copy will have nonzero curvature. Since three dimensional gravity does not propagate, this implies that the double copy of a localized charge density in three dimensions cannot be a vacuum solution, but rather the gravitational source must permeate spacetime in such a way as to mimic the Newtonian behavior of higher-dimensional gravity. In what follows we present an explicit example of such a system.

\subsection{Point charge in $2+1$ dimensions and its double copy}

As a specific example of the Kerr-Schild double copy of a given gauge field solution, we now consider a static point charge sitting at the origin. The corresponding gauge field can be written as

$$
A_{\mu}=k_{\mu} \phi, \quad k_{\mu} \mathrm{d} x^{\mu}=-\mathrm{d} t-\mathrm{d} r, \quad \phi=-\frac{g Q}{2 \pi} \log r,
$$

using polar coordinates $(t, r, \theta)$. One may explicitly verify that the gauge field $A^{\mu}$ satisfies the Maxwell equations $\nabla_{\nu} F^{\mu \nu}=J^{\mu}$ with a source current

$$
J^{\mu} \partial_{\mu}=g Q \delta^{2}(r) \partial_{t}
$$

using the fact that $\vec{\nabla}^{2} \log r=2 \pi \delta^{2}(r)$ in two spatial dimensions. Strictly speaking, we should introduce a scaling factor $r_{0}$ into $\phi$ so as to make the logarithm dimensionless. However, we may choose units such that this is unity for what follows. Notice that the vector $k$ is null and geodesic as required, ${ }^{6}$ and $\phi$ satisfies the linearized (static) equations of motion of the biadjoint scalar. Given this, we are able to construct the classical double copy of the static charge. The double copy is given by the Kerr-Schild metric

$$
g_{\mu \nu}=\eta_{\mu \nu}+\kappa k_{\mu} k_{\nu} \phi=\left(\begin{array}{ccc}
-1-\frac{\kappa^{2} M}{4 \pi} \log r & -\frac{\kappa^{2} M}{4 \pi} \log r & 0 \\
-\frac{\kappa^{2} M}{4 \pi} \log r & 1-\frac{\kappa^{2} M}{4 \pi} \log r & 0 \\
0 & 0 & r^{2}
\end{array}\right),
$$

where we have made the standard replacements [52]

$$
g \rightarrow \frac{\kappa}{2}, \quad Q \rightarrow M .
$$

Substituting the metric of eq. (5.7) into the Einstein's equations, we find a non-zero stress tensor given by

$$
T^{\mu}{ }_{\nu}=-\frac{1}{\kappa}\left(\begin{array}{ccc}
\frac{\phi^{\prime}}{r} & 0 & 0 \\
0 & \frac{\phi^{\prime}}{r} & 0 \\
0 & 0 & \phi^{\prime \prime}
\end{array}\right)=\frac{M}{4 \pi r^{2}}\left(\begin{array}{ccc}
1 & 0 & 0 \\
0 & 1 & 0 \\
0 & 0 & -1
\end{array}\right) .
$$

It is interesting already to note that, although the gauge field source is localized to the origin, the gravitational source is not. This is possible due to the relation between the

\footnotetext{
${ }^{6}$ Our gauge field, which has nonzero spatial components, is related to the perhaps more intuitive solution $A^{0}=-\frac{g Q}{2 \pi} \log r, A^{i}=0$ by a gauge transformation, which is necessary in order for $k$ to satisfy these requirements. (See the previous footnote about the gauge-dependence of the double copy.)
} 
sources given in eq. (1.6), and indeed one can easily see that this relation holds: using the first expression for the stress tensor in eq. (5.9) leads to a gauge theory source current

$$
J^{\mu} \partial_{\mu}=\left(-\vec{\nabla}^{2} \phi, 0,0\right)=g Q \delta^{2}(r)(1,0,0),
$$

as required. We have therefore found an explicit example of the conclusion of the previous section, namely that the double copy of a localised gauge theory source cannot correspond to a vacuum solution of the Einstein equations in $d=3$.

The presence of a non-trivial source term implies the presence of matter degrees of freedom. This may at first sight seem puzzling, until we remember that the double copy of pure gauge theory is not pure gravity, but gravity coupled to a dilaton and two-form. The latter will be absent, as in previous examples [52-54,68], due to the symmetric nature of the double copied field. However, the dilaton may indeed be turned on, and thus we would like to identify the above stress-energy tensor with a non-zero dilaton field. In order to do so, we consider a free massless scalar field. Such a field is invariant under the symmetry

$$
\varphi \rightarrow \pm \varphi+c
$$

and its stress-energy tensor reads

$$
T_{\nu}^{\mu}=\partial^{\mu} \varphi \partial_{\nu} \varphi-\frac{1}{2} g_{\nu}^{\mu}(\partial \varphi)^{2}
$$

The Einstein equations then imply a Ricci tensor

$$
R_{\nu}^{\mu}=\kappa^{2} / 2 \partial^{\mu} \varphi \partial_{\nu} \varphi
$$

On the other hand, from the metric in eq. (5.7) we find

$$
R^{\mu}{ }_{\nu}=-\frac{\kappa^{2} M}{4 \pi r^{2}}\left(\begin{array}{lll}
0 & 0 & 0 \\
0 & 0 & 0 \\
0 & 0 & 1
\end{array}\right) .
$$

Comparing these results, we obtain that the dilaton profile must be linear in the azimuthal angle. Furthermore, the difference in sign between eqs. (5.12) and (5.13) implies that either the scalar field is imaginary, or that it has the wrong sign in its kinetic term. The second option in fact makes sense, albeit for a rather complicated reason. For amplitudes, the double copy is known to be true only in certain generalised gauges, where one has potentially performed both a gauge transformation and a field redefinition. It was known already before the double copy [48] that it is possible to make field redefinitions so as to make the gravitational Lagrangian consistent with the KLT relations [14] relating gauge and gravity amplitudes at tree-level (and which are equivalent to the double copy). That is, by introducing a dilaton and starting in the de Donder gauge, one may transform the graviton and dilaton fields so as to remove all the terms in the graviton Lagrangian that mix left and right indices, leaving a structure that is manifestly double-copy-like. This turns out to have the side-effect of reversing the sign of the dilaton kinetic term. Returning to our present study of classical solutions, we should then expect that if the double copy of a 
given gauge field generates a non-zero dilaton profile, the latter should be associated with a "wrong-sign" dilaton kinetic term. The fact that this has not been noticed before is simply because previous examples of the Kerr-Schild double copy involve zero dilaton profiles on the gravity side [52-54,68]. Nevertheless, the fact that the double copy does not satisfy certain energy conditions has been previously observed [56].

From the above results, the explicit form of the dilaton for the double copy of the point charge in $d=3$ is defined by ${ }^{7}$

$$
\partial_{\mu} \varphi=\sqrt{M / 2 \pi}(0,0,1)
$$

The identification of the source term in eq. (5.9) with a nonzero dilaton profile is natural, given that a similar matching of gauge theory degrees of freedom with the dilaton can be made for the plane wave states that enter the original BCJ double copy for scattering amplitudes. However, we may still worry whether or not this is the only possible interpretation of eq. (5.9). To this end, two remarks are useful. Firstly, we may check the equation of motion of the dilaton. Considering the field profile from eq. (5.14) we see that it indeed satisfies

$$
\nabla^{\mu} \nabla_{\mu} \varphi=0
$$

where $\nabla_{\mu}$ is the covariant derivative of the metric (5.7). Secondly, we have verified that the stress tensor from eq. (5.9) cannot correspond to that of a standard viscous fluid, which reinforces its dilaton interpretation; for details on this calculation see appendix A. In the following subsections, we analyze different aspects of this dilaton-gravity solution which will strengthen its interpretation as the double copy of a static charge in $(2+1)$ dimensions.

\subsubsection{Newtonian-like limit}

In this section, we examine further aspects of the Newtonian-like limit of our gravity-dilaton system. First, we recall that the trajectory $x^{\mu}(t)$ of a particle interacting with Newtonian gravity in $(2+1)$ dimensions satisfies

$$
\frac{\mathrm{d}^{2} \boldsymbol{x}}{\mathrm{d} t}=-\vec{\nabla} \Phi, \quad \vec{\nabla}^{2} \Phi=2 \pi G \rho,
$$

where $\Phi$ is the Newtonian potential, $\rho$ is the mass density, and $G$ is Newton's constant. Outside a spherical source the Newtonian potential is given by $\Phi=G M \log r$. On the other hand, the Newtonian limit of General Relativity in $d$ dimensions gives rise to

$$
\frac{\mathrm{d}^{2} \boldsymbol{x}}{\mathrm{d} t^{2}}=-\vec{\nabla} \Phi, \quad \vec{\nabla}^{2} \Phi=\frac{\kappa^{2}}{2} \rho\left(\frac{d-3}{d-2}\right),
$$

as in eqs. (3.7), (3.9), which as previously mentioned shows that in $(2+1)$ dimensions masses do not experience Newtonian forces.

\footnotetext{
${ }^{7}$ The reader may worry that the form of the dilaton itself is linear in the azimuthal angle, and thus not periodic. However, the Einstein equations imply that eq. (5.14) need only be satisfied locally. A global dilaton profile can be obtained by patching solutions together, up to transformations of the form of eq. (5.10).
} 
There have been some attempts at reproducing the expected Newtonian limit in scalartensor theories. For example, a solution with a logarithmic Newtonian potential at short distances was found in [90]. There, a scalar-tensor theory in $(2+1)$ dimensions was formulated as a dimensional reduction of a $4 \mathrm{~d}$ theory, by taking solutions with no dependence in a particular direction. Similar approaches have also been examined in e.g. ref. [91]. In this paper, we have seen that the Kerr-Schild double copy naturally generates a scalartensor theory that mimics the presence of Newtonian gravity: eq. (5.4) relates the relevant Newtonian potential to the gravitational source generated by double-copying a given gauge field, where the source is associated with a nonzero dilaton.

Let us now consider the Newtonian limit of the explicit dilaton-gravity solution found above. This limit is in fact not Newtonian in the strictest sense: we assume nonrelativistic trajectories, so that we obtain $\mathrm{d}^{2} \boldsymbol{x} / \mathrm{d} t^{2}=-\vec{\nabla} \Phi$ with $\Phi=-\frac{1}{2} h_{00}$, but we do not take the usual Newtonian limit of the source, since doing so requires that the source term $\frac{\kappa^{2}}{2}\left(T-T_{0}^{0}\right)$ for $\Phi$ vanish. Indeed, if we just leave the gravitational source alone and use eq. (5.9) we find

$$
\vec{\nabla}^{2} \Phi=\frac{\kappa^{2}}{4} M \delta^{2}(r)
$$

from which we deduce the non-point-like form of the stress-energy tensor (5.9) is such that the corresponding Newtonian potential has a pointlike source. Further, from the Newtonian potential

$$
\Phi=\frac{\kappa^{2} M}{8 \pi} \log r
$$

we can read off Newton's constant for our system:

$$
G=\frac{\kappa^{2}}{8 \pi} \text {. }
$$

In obtaining eq. (5.18) we did not need to make any assumptions about the source, since from the mixed-index stress-energy tensor (5.9) we can just take the trace and evaluate. However from this equation it is clear that the appropriate limit to take is the one in which the parameter $G M$, which multiplies all diagonal elements of the stress-energy tensor, is small. This corresponds to taking the charge $Q$ of the point source on the gauge side to be small, but does not correspond to a small point mass on the gravity side, since the gravitational source is nonzero throughout spacetime. But by the arguments of section 5.1 and eq. (5.18) we see that this is in fact the correct limit to take if we wish to recover a Newtonian limit with a nonvanishing pointlike source, which requires a stress-energy tensor which is nonvanishing throughout spacetime.

In simple terms, the lack of a propagating graviton upon taking the double copy forces the dilaton to turn on, in order to match up with the physical degrees of freedom in the gauge theory. This then ensures a well-behaved Newtonian-like limit, in accordance with the fact that there is a well-behaved Newtonian limit in the gauge theory. Note that this correspondence also allows us to interpret the parameter $M$ in the gravity solution: it is the mass one would obtain in Newtonian gravity. As in the Schwarzschild example of ref. [52], electric charge in the gauge theory then maps to mass in the gravity theory, provided a suitable definition is used for the latter. 


\subsubsection{Orbits and perihelion precession}

To gain yet more physical insight into our solution, it is instructive to examine the behaviour of geodesics corresponding to the motion of (massive) test particles. To this end, notice that the metric of eq. (5.7) can be written in "Schwarzschild" coordinates by performing the coordinate transformation

$$
\mathrm{d} t=\mathrm{d} T+\left((1+2 G M \log r)^{-1}-1\right) \mathrm{d} r,
$$

in which coordinates the metric becomes

$$
\mathrm{d} s^{2}=-(1+2 G M \log r) \mathrm{d} T^{2}+(1+2 G M \log r)^{-1} \mathrm{~d} r^{2}+r^{2} \mathrm{~d} \phi^{2} .
$$

In these coordinates the geodesic equation for timelike observers can be written as

$$
\frac{1}{2} E^{2}=\frac{1}{2}\left(\frac{\mathrm{d} r}{\mathrm{~d} T}\right)^{2}+V_{\mathrm{eff}}, \quad V_{\mathrm{eff}}=\frac{1}{2}\left(\frac{L^{2}}{r^{2}}+1\right)\left(1+2 G M \log r / r_{0}\right),
$$

where we have reintroduced the scale $r_{0}$ i.e. a constant radius whose value is set by boundary conditions. In the expression above, we have used the conserved quantities related to the timelike $\xi_{t}$ and angular $\xi_{\theta}$ Killing vectors:

$$
E=-g_{\mu \nu} \xi_{t}^{\mu} u^{\mu}, \quad L=g_{\mu \nu} \xi_{\theta}^{\mu} u^{\mu}
$$

where $u^{\mu}$ is a unit timelike vector tangent to the geodesic. Physically, $E$ and $L$ represent the energy and angular momentum (relative to the origin) of the test particle. In eq. (5.22), we have introduced the so-called effective potential $V_{\text {eff. }}$. In the Newtonian limit, a similar geodesic equation is obtained, but where the effective potential is replaced by

$$
V_{\text {Newt }}=\frac{1}{2}+\frac{L^{2}}{2 r^{2}}+G M \log r / r_{0},
$$

which we obtain from eq. (5.22) by neglecting the $L^{2} G M$ term. As is suggested by the form of eq. (5.22), values of the parameters $\left(E, r_{0}, L\right)$ exist such that timelike geodesics form closed orbits. We may find the relevant conditions by examining the form of the effective potential, which is plotted for various values of $L$ in figure 1 . We see that the Newtonian potential always has an infinite barrier at short distances, whilst the GR result turns over at a finite distance. Furthermore (and in marked contrast to the case of the Schwarszchild black hole in four spacetime dimensions), a timelike observer can never escape to infinity, due to the fact that the effective potential is logarithmically divergent there. This can be traced back to the logarithmic behaviour of the gauge theory propagator in two spatial dimensions, which enters the effective potential via the double copy.

Examining the GR effective potential in more detail, we find that it has a maximum as long as

$$
L>L_{\min } \equiv r_{0} e^{1-\frac{1}{2 G M}} .
$$

We will therefore impose this constraint in order to obtain stable orbits. In the same spirit, we will restrict the energy range such that

$$
V_{\text {eff }}^{\min }<E<V_{\text {eff }}^{\max }
$$



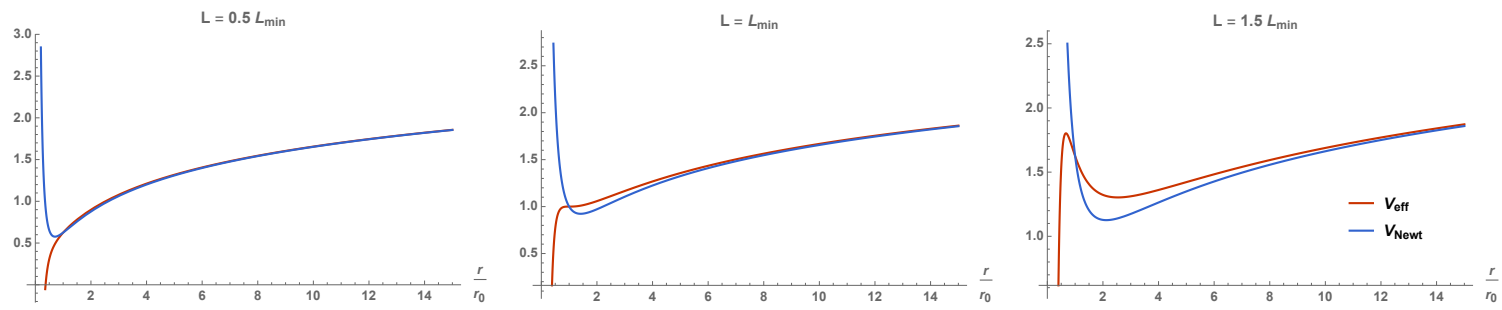

Figure 1. Effective GR and Newtonian potentials for $G M=1 / 2$ and different values of the angular momentum $L$. We can clearly observe that for $L<L_{\min }$ the GR potential has no maximum, at $L=L_{\min }$ it develops an inflection point, and for $L>L_{\min }$ a maximum occurs and we can have stable orbits. On the other hand, the shape of the Newtonian potential stays unchanged and always allows stable orbits.

where the maximum of the potential can be approximated for $L \gg r_{0}$ as follows:

$$
V_{\mathrm{eff}}^{\max } \simeq \frac{1}{2} G M\left(\frac{L}{r_{0}}\right)^{2} e^{\frac{1}{G M}-1} .
$$

Meanwhile, we can only find an analytic expression for the minimum of the potential in the Newtonian limit where $G M L^{2} / r^{2} \ll 1$, yielding

$$
V_{\mathrm{eff}}^{\min } \simeq \frac{1}{2}+\frac{1}{2} G M+G M \log \left(\frac{L}{\sqrt{G M} r_{0}}\right)
$$

In order to visualise the orbits we must solve for $r(\theta)$. By using the fact that $\dot{\theta}=L / r^{2}$, where the dot indicates a derivative with respect to $T$ and $\theta$ is the azimuthal angle, we get

$$
\left(\frac{\mathrm{d} r}{\mathrm{~d} \theta}\right)^{2}-\frac{E^{2} r^{4}}{L^{2}}+\frac{r^{4}}{L^{2}}+\frac{r^{4} 2 G M \log r}{L^{2}}+r^{2}+r^{2} 2 G M \log r=0,
$$

To further analyze this equation, it is convenient to change variables to the dimensionless quantity $x=L / G M r$, and apply a derivative with respect to $\theta$ (denoted by a prime in what follows):

$$
G M x^{\prime \prime}+G^{2} M^{2}\left(2 x \log \left(\frac{L}{G M x}\right)-x\right)+G M x-\frac{1}{x}=0 .
$$

Taking the limit where $G M \ll 1$ we obtain

$$
x^{\prime \prime}+x-\frac{1}{G M x}=0,
$$

which indeed corresponds to the expected Newtonian limit given by the potential in eq. (5.19). In both the GR and Newtonian cases, we may solve the radial equation numerically, and plot the orbits in the $(r, \theta)$ plane. Considering first the GR case, we find qualitatively different solutions, according to the value of the quantity

$$
\alpha=\frac{L^{2}}{r_{0}^{2}} G M
$$



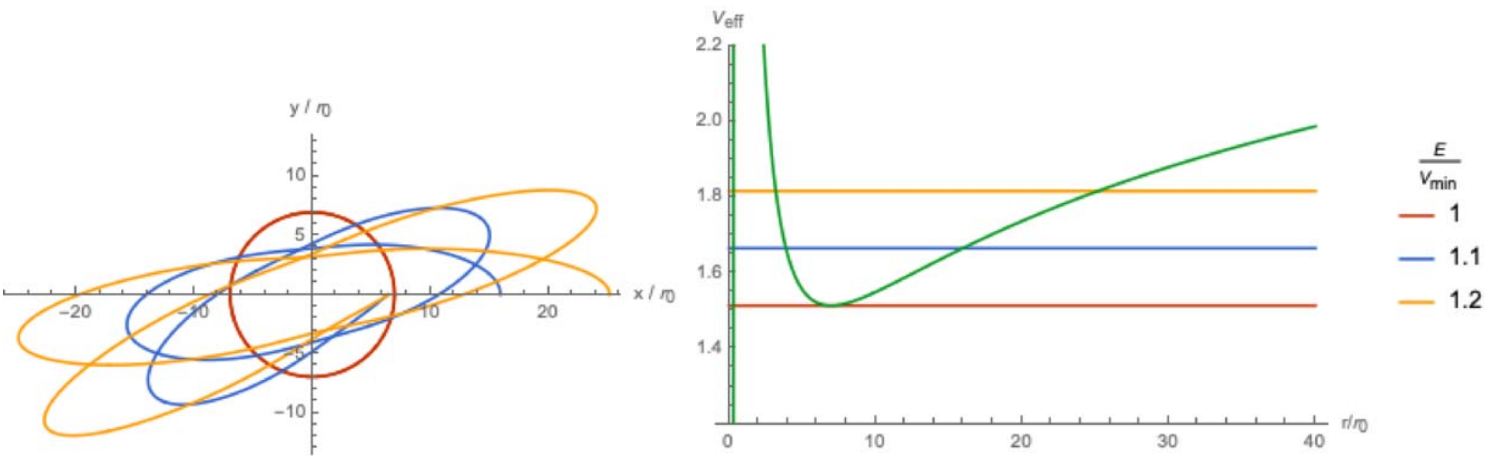

Figure 2. Timelike geodesics obtained for the GR effective potential, where $G M=0.4$ and $L / r_{0}=3$.
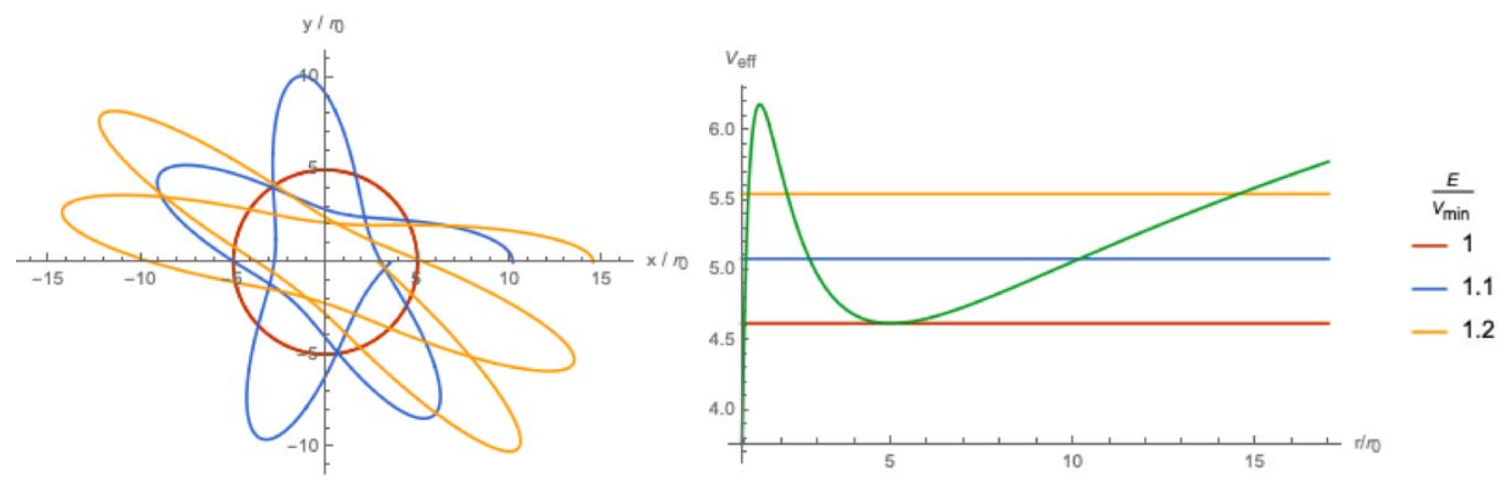

Figure 3. Timelike geodesics obtained for the GR effective potential, where $G M=1.8$ and $L / r_{0}=3$.

For small values of $\alpha$, one obtains a circular orbit if $E=V_{\min }$, and a precessing elliptical orbit otherwise, as shown in figure 2. Alternatively, for sufficiently high values of $\alpha$ we find that the orbits have an interesting "starfish" shape, owing to the increasingly repulsive nature of the potential barrier at small radial distances. This is shown in figure 3 . In both figures, we also show the form of the effective potential in each case, with colour-coded values for the energy $E$.

The phenomenon of precession is common to the case of the Schwarzschild metric in $d=4$, as indeed it must be: Bertrand's theorem states that the only central forces that give rise to stable non-precessing orbits are an inverse square law, or a linear radial dependence characteristic of a harmonic oscillator. The GR effective potential in the present case is neither, and thus we expect to see precession. A further consequence of this theorem, however, is that we also expect to see precession in the Newtonian case, unlike the case of Newtonian gravity in $d=4$ (i.e. the latter obeys an inverse square force law). This is shown in figure 4, where we also show an orbit obtained from the full GR potential for identical initial conditions. We see that switching from the Newtonian theory to the full GR case enhances the precession, analogous to the Schwarzschild case. 


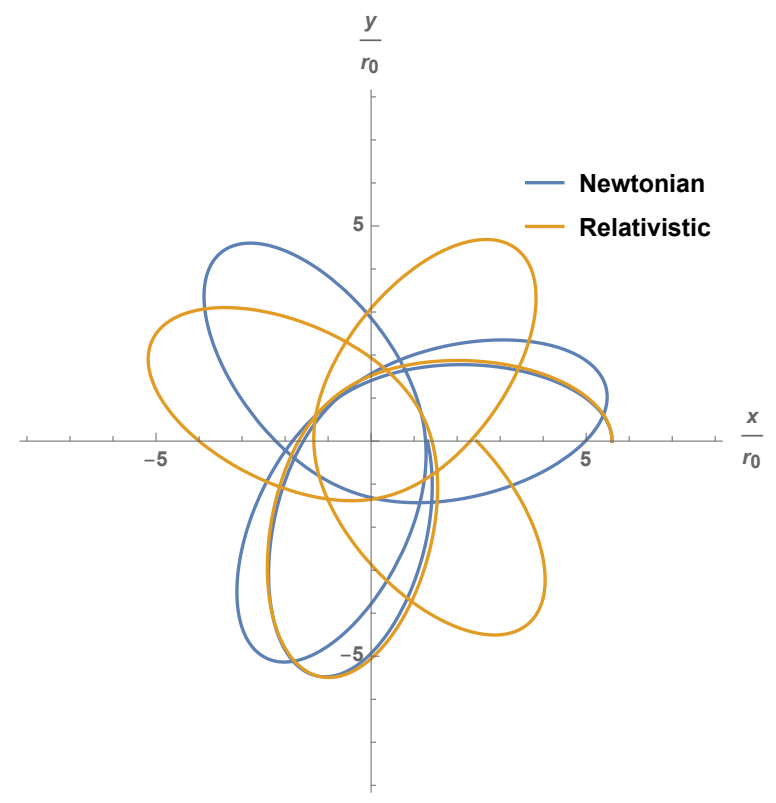

Figure 4. Bounded orbits obtained via solving Eqns (5.30) (displayed as the blue line) and (5.29) (displayed as the orange line) with $G M=.2, L / r_{0}=1$, and $E=1.1 V_{\min }$.

\section{Conclusion}

In this paper, we have examined the double copy of exact classical solutions in $(2+1)$ dimensions. This procedure has been defined for any time-dependent Kerr-Schild solution in gravity [52] and studied in a number of non-trivial examples [53, 54, 68], but poses unique conceptual puzzles in three spacetime dimensions. In particular, three dimensional gravity in the form of pure General Relativity is known to have no propagating degrees of freedom, and also lacks a sensible Newtonian limit. It is then not clear how physical degrees of freedom in the gauge theory can be matched to the gravity theory in a consistent manner.

We first reviewed an existing analysis [81] of a pure gravity-like solution, namely the BTZ black hole [87], whose Kerr-Schild form was first presented in ref. [88]. Upon taking the single copy, the only surviving remnant in the gauge theory is a constant charge density filling all space, which corresponds with the cosmological constant (a constant energy density) in the gravity theory, in accordance with the four-dimensional results of ref. [53].

Next, we considered a pointlike electric charge in (2+1)-dimensional gauge theory, for which the Kerr-Schild double copy is straightforward. This turned out to yield a nonvacuum solution of the Einstein equations, in accordance with general observations we made that localised sources in gauge theory cannot lead to vacuum solutions under the Kerr-Schild double copy. The source term on the gravity side could be interpreted as a non-zero dilaton profile, which is consistent with the fact that the double copy of pure gauge theory is not General Relativity, but rather GR coupled to a dilaton and two-form. This is itself highly novel, in that our example is the first case of the Kerr-Schild double copy in which the dilaton is non-zero on the gravity side of the correspondence. Furthermore, it resolves the issue of how to map the physical degrees of freedom in the gauge theory: with 
no propagating graviton, the dilaton has to turn on to do gravity's job. This in turn leads to a well-behaved Newtonian-like limit, such that charge in the gauge theory maps to mass on the gravity side, where the mass parameter can be interpreted by consistency with the correct gravitational potential in the Newtonian limit. This construction is reminiscent of previous attempts to obtain sensible Newtonian limits in various scalar-tensor theories [90, 91]. To physically illustrate our analysis, we examined timelike geodesics in the dilatongravity system obtained by copying the point charge. These consist (for appropriate initial conditions) of stable, precessing orbits. The Newtonian orbits also precess in $d=3$, but the effect in the GR case is exacerbated, analogous to the case of $d=4$.

An outstanding property of this dilaton-gravity solution obtained as the double copy is the existence of an event horizon at

$$
r_{h}=r_{0} e^{-\frac{1}{2 G M}}
$$

which can be observed from the metric in Schwarzschild coordinates in eq. (5.21). This implies that the solution corresponds to a phantom black hole. In the present case, the no-hair theorem [92-94] is avoided since the scalar field is a ghost and it does not inherit the symmetries of the spacetime, that is, $\partial_{\theta} \varphi \neq 0$. Phantom black holes and their thermodynamic properties have previously been studied, for example in [95, 96]. It would be interesting to study the thermodynamic properties of this 3-dimensional phantom black hole. Another interesting direction is to consider time dependent solutions for the dilaton and also including additional fields and matter. We leave these ideas to future work.

The study of the double copy for classical solutions is still in its infancy, and useful insights can be gained by examining highly unusual cases, even if these are not directly physical by themselves. We hope that our results prove useful in this regard, and stimulate further work in this area.

\section{Acknowledgments}

CDW was supported by the Science and Technology Facilities Council (STFC) Consolidated Grant ST/P000754/1 "String theory, gauge theory \& duality", and by the European Union's Horizon 2020 research and innovation programme under the Marie SkłodowskaCurie grant agreement No. 764850 "SAGEX". The research of BM, KR, and SW was supported in part by DOE grant DE-FG02-85ER40237. The work of M.C.G. was supported in part by US Department of Energy (HEP) Award DE-SC0013528. We would also like to thank the referee for comments that led to improvements to the original draft.

\section{A No fluid interpretation of the double copy stress-energy tensor}

In section 5.2, we have seen that the stress-energy tensor generated by double-copying a gauge theory point charge (eq. (5.9)) in $(2+1)$ dimensions can be associated with a dilaton profile. In this appendix, we ask whether it is possible to instead associate this with a viscous fluid. For the latter, the stress tensor has the generic form

$$
T_{\mu \nu}=\rho u_{\mu} u_{\nu}+P \gamma_{\mu \nu}-\zeta \theta \gamma_{\mu \nu}+2 \eta \sigma_{\mu \nu}+\Pi^{\rho} \gamma_{\rho(\mu} u_{\nu)} .
$$


where $\rho$ is the energy density, $P$ the pressure, $\eta$ the shear viscosity, $\zeta$ the bulk viscosity, $\theta \equiv \gamma^{\nu \mu} \nabla_{\nu} u_{\mu}$ the expansion, and $\Pi^{\rho}$ the momentum density. The metric of the surface perpendicular to the four velocity $u^{\mu}$ is

$$
\gamma_{\mu \nu}=g_{\mu \nu}+u_{\mu} u_{\nu}
$$

where the four velocity is normalized such that $u \cdot u=-1$. Lastly, the shear tensor $\sigma_{\mu \nu}$ is

$$
\sigma_{\mu \nu} \equiv \frac{1}{2}\left(\gamma_{\mu}^{\rho} \nabla_{\rho} u_{\nu}+\gamma_{\nu}^{\rho} \nabla_{\rho} u_{\mu}\right)-\frac{1}{2} \gamma_{\mu \nu} \theta
$$

In vacuum $(2+1)$ dimensional gravity there is no shear, but the situation can change in the presence of matter.

We will now assume that the stress tensor for the double copy of the static charge can be written as in eq. (A.1). With this assumption, the fluid properties can be extracted from the stress tensor as

$$
\begin{aligned}
\rho & =T_{\mu \nu} u^{\mu} u^{\nu} \\
2(P-\zeta \theta) & =T_{\mu \nu} \gamma_{\rho}^{\mu} \gamma^{\nu \rho} \\
\Pi^{\rho} & =T_{\mu \nu} \gamma^{\rho \nu} u^{\mu}
\end{aligned}
$$

Working in Kerr-Schild coordinates, that is, with the metric given by eq. (5.7), we can see that the four velocity is given by

$$
u^{\mu}=\frac{1}{\sqrt{1+4 G M \log (r)}}(1,0,0)
$$

and thus

$$
\rho=-\frac{M}{4 \pi r^{2}}, \quad P-\zeta \theta=P=0, \quad \Pi^{\rho}=(0,0,0), \quad \sigma_{\mu \nu}=\mathbf{0} .
$$

From these, we see that the covariant derivative of the four-velocity can be written as

$$
\nabla_{\mu} u_{\nu}=-\dot{u}_{\mu} u_{\nu}
$$

This describes changes in the relative position of the worldlines of two neighboring timelike observers. Note that the shear, vorticity, and expansion are zero. Thus, the shape of the cross-sectional area orthogonal to the timelike geodesic flow is not deformed as we move along the geodesics. This behavior is specific for the congruence constructed above, but in general it could be possible to find cases where the area orthogonal to the flow is deformed.

Using the above result, we can find a discrepancy in the assumption that the stress tensor of eq. (5.9) is the stress tensor of a viscous fluid with four-velocity given by eq. (A.7). While for a fluid with the properties in eq. (A.8) we have

$$
T_{\rho \lambda}^{\text {fluid }} \gamma_{\mu}^{\rho} \gamma_{\nu}^{\lambda}=P \gamma_{\mu \nu}-\zeta \theta \gamma_{\mu \nu}+2 \eta \sigma_{\mu \nu}=\mathbf{0}
$$

for the stress tensor in eq. (5.9) we have

$$
\Sigma_{\mu \nu} \equiv T_{\rho \lambda} \gamma_{\mu}^{\rho} \gamma_{\nu}^{\lambda}=\frac{M}{4 \pi}\left(\begin{array}{ccc}
0 & 0 & 0 \\
0 & 1 & 0 \\
0 & 0 & -1
\end{array}\right)
$$


Thus, the stress tensor of the double copy can be written as

$$
T_{\mu \nu}=\rho u_{\mu} u_{\nu}+\Sigma_{\mu \nu} .
$$

Since $\Sigma_{\mu \nu}$ cannot be interpreted as a property of a fluid, this implies that the stress tensor of eq. (5.9) does not correspond to a viscous fluid. Note that we could identify the stress tensor in eq. (5.9) with that of a "Euclidean" perfect fluid if we consider a spacelike fourvelocity. This is easily seen by considering $u^{\mu} \propto \partial^{\mu} \varphi$ where $\partial^{\mu} \varphi$ is given by eq. (5.14).

Open Access. This article is distributed under the terms of the Creative Commons Attribution License (CC-BY 4.0), which permits any use, distribution and reproduction in any medium, provided the original author(s) and source are credited.

\section{References}

[1] Z. Bern, J.J.M. Carrasco and H. Johansson, New relations for gauge-theory amplitudes, Phys. Rev. D 78 (2008) 085011 [arXiv: 0805.3993] [INSPIRE].

[2] J. Broedel and L.J. Dixon, Color-kinematics duality and double-copy construction for amplitudes from higher-dimension operators, JHEP 10 (2012) 091 [arXiv:1208.0876] [INSPIRE].

[3] S. He and Y. Zhang, New formulas for amplitudes from higher-dimensional operators, JHEP 02 (2017) 019 [arXiv: 1608.08448] [INSPIRE].

[4] M. Cofano, C.-H. Fu and K. Krasnov, Deformations of Yang-Mills theory, Phys. Rev. D 92 (2015) 065012 [arXiv:1501.00848] [INSPIRE].

[5] N.E.J. Bjerrum-Bohr, P.H. Damgaard and P. Vanhove, Minimal basis for gauge theory amplitudes, Phys. Rev. Lett. 103 (2009) 161602 [arXiv:0907.1425] [INSPIRE].

[6] S. Stieberger, Open \& closed vs. pure open string disk amplitudes, arXiv:0907.2211 [INSPIRE].

[7] Z. Bern, T. Dennen, Y.-T. Huang and M. Kiermaier, Gravity as the square of gauge theory, Phys. Rev. D 82 (2010) 065003 [arXiv: 1004.0693] [InSPIRE].

[8] N.E.J. Bjerrum-Bohr, P.H. Damgaard, T. Sondergaard and P. Vanhove, Monodromy and Jacobi-like relations for color-ordered amplitudes, JHEP 06 (2010) 003 [arXiv:1003.2403] [INSPIRE].

[9] B. Feng, R. Huang and Y. Jia, Gauge amplitude identities by on-shell recursion relation in S-matrix program, Phys. Lett. B 695 (2011) 350 [arXiv: 1004.3417] [INSPIRE].

[10] S.H. Henry Tye and Y. Zhang, Dual identities inside the gluon and the graviton scattering amplitudes, JHEP 06 (2010) 071 [Erratum ibid. 04 (2011) 114] [arXiv:1003.1732] [INSPIRE].

[11] C.R. Mafra, O. Schlotterer and S. Stieberger, Explicit BCJ numerators from pure spinors, JHEP 07 (2011) 092 [arXiv:1104.5224] [INSPIRE].

[12] R. Monteiro and D. O'Connell, The kinematic algebra from the self-dual sector, JHEP 07 (2011) 007 [arXiv: 1105.2565] [INSPIRE].

[13] N.E.J. Bjerrum-Bohr, P.H. Damgaard, R. Monteiro and D. O'Connell, Algebras for amplitudes, JHEP 06 (2012) 061 [arXiv: 1203.0944] [INSPIRE]. 
[14] H. Kawai, D.C. Lewellen and S.H.H. Tye, A relation between tree amplitudes of closed and open strings, Nucl. Phys. B 269 (1986) 1 [INSPIRE].

[15] Z. Bern, J.J.M. Carrasco and H. Johansson, Perturbative quantum gravity as a double copy of gauge theory, Phys. Rev. Lett. 105 (2010) 061602 [arXiv: 1004.0476] [INSPIRE].

[16] Z. Bern, L.J. Dixon, D.C. Dunbar, M. Perelstein and J.S. Rozowsky, On the relationship between Yang-Mills theory and gravity and its implication for ultraviolet divergences, Nucl. Phys. B 530 (1998) 401 [hep-th/9802162] [INSPIRE].

[17] M.B. Green, J.H. Schwarz and L. Brink, $N=4$ Yang-Mills and $N=8$ supergravity as limits of string theories, Nucl. Phys. B 198 (1982) 474 [inSPIRE].

[18] Z. Bern, J.S. Rozowsky and B. Yan, Two loop four gluon amplitudes in $N=4$ super Yang-Mills, Phys. Lett. B 401 (1997) 273 [hep-ph/9702424] [INSPIRE].

[19] J.J. Carrasco and H. Johansson, Five-point amplitudes in $N=4$ super-Yang-Mills theory and $N=8$ supergravity, Phys. Rev. D 85 (2012) 025006 [arXiv:1106.4711] [INSPIRE].

[20] J.J.M. Carrasco, M. Chiodaroli, M. Günaydin and R. Roiban, One-loop four-point amplitudes in pure and matter-coupled $N \leq 4$ supergravity, JHEP 03 (2013) 056 [arXiv:1212.1146] [INSPIRE].

[21] C.R. Mafra and O. Schlotterer, The structure of n-point one-loop open superstring amplitudes, JHEP 08 (2014) 099 [arXiv: 1203.6215] [INSPIRE].

[22] R.H. Boels, R.S. Isermann, R. Monteiro and D. O'Connell, Colour-kinematics duality for one-loop rational amplitudes, JHEP 04 (2013) 107 [arXiv: 1301.4165] [INSPIRE].

[23] N.E.J. Bjerrum-Bohr, T. Dennen, R. Monteiro and D. O'Connell, Integrand oxidation and one-loop colour-dual numerators in $N=4$ gauge theory, JHEP 07 (2013) 092 [arXiv: 1303.2913] [INSPIRE].

[24] Z. Bern, S. Davies, T. Dennen, Y.-T. Huang and J. Nohle, Color-kinematics duality for pure Yang-Mills and gravity at one and two loops, Phys. Rev. D 92 (2015) 045041 [arXiv: 1303.6605] [INSPIRE].

[25] Z. Bern, S. Davies and T. Dennen, The ultraviolet structure of half-maximal supergravity with matter multiplets at two and three loops, Phys. Rev. D 88 (2013) 065007 [arXiv:1305.4876] [INSPIRE].

[26] J. Nohle, Color-kinematics duality in one-loop four-gluon amplitudes with matter, Phys. Rev. D 90 (2014) 025020 [arXiv:1309.7416] [INSPIRE].

[27] Z. Bern, S. Davies, T. Dennen, A.V. Smirnov and V.A. Smirnov, Ultraviolet properties of $N=4$ supergravity at four loops, Phys. Rev. Lett. 111 (2013) 231302 [arXiv:1309.2498] [INSPIRE].

[28] S.G. Naculich, H. Nastase and H.J. Schnitzer, All-loop infrared-divergent behavior of most-subleading-color gauge-theory amplitudes, JHEP 04 (2013) 114 [arXiv:1301.2234] [INSPIRE].

[29] Y.-J. Du, B. Feng and C.-H. Fu, Dual-color decompositions at one-loop level in Yang-Mills theory, JHEP 06 (2014) 157 [arXiv:1402.6805] [INSPIRE].

[30] C.R. Mafra and O. Schlotterer, Towards one-loop SYM amplitudes from the pure spinor BRST cohomology, Fortsch. Phys. 63 (2015) 105 [arXiv:1410.0668] [INSPIRE]. 
[31] Z. Bern, S. Davies and T. Dennen, Enhanced ultraviolet cancellations in $N=5$ supergravity at four loops, Phys. Rev. D 90 (2014) 105011 [arXiv: 1409.3089] [InSPIRE].

[32] C.R. Mafra and O. Schlotterer, Two-loop five-point amplitudes of super Yang-Mills and supergravity in pure spinor superspace, JHEP 10 (2015) 124 [arXiv: 1505.02746] [INSPIRE].

[33] S. He, R. Monteiro and O. Schlotterer, String-inspired BCJ numerators for one-loop MHV amplitudes, JHEP 01 (2016) 171 [arXiv:1507.06288] [INSPIRE].

[34] Z. Bern, S. Davies and J. Nohle, Double-copy constructions and unitarity cuts, Phys. Rev. D 93 (2016) 105015 [arXiv: 1510.03448] [INSPIRE].

[35] G. Mogull and D. O'Connell, Overcoming obstacles to colour-kinematics duality at two loops, JHEP 12 (2015) 135 [arXiv:1511.06652] [INSPIRE].

[36] M. Chiodaroli, M. Günaydin, H. Johansson and R. Roiban, Spontaneously broken Yang-Mills-Einstein supergravities as double copies, JHEP 06 (2017) 064 [arXiv: 1511.01740] [INSPIRE].

[37] Z. Bern, J.J.M. Carrasco, W.-M. Chen, H. Johansson, R. Roiban and M. Zeng, Five-loop four-point integrand of $N=8$ supergravity as a generalized double copy, Phys. Rev. D 96 (2017) 126012 [arXiv:1708.06807] [INSPIRE].

[38] H. Johansson and A. Ochirov, Color-kinematics duality for QCD amplitudes, JHEP 01 (2016) 170 [arXiv: 1507.00332] [INSPIRE].

[39] J.J.M. Carrasco, Gauge and gravity amplitude relations, in Proceedings, Theoretical Advanced Study Institute in Elementary Particle Physics: Journeys Through the Precision Frontier: Amplitudes for Colliders (TASI 2014), Boulder, CO, U.S.A., 2-27 June 2014, World Scientific, Singapore (2015), pg. 477 [arXiv: 1506.00974] [INSPIRE].

[40] S. Oxburgh and C.D. White, BCJ duality and the double copy in the soft limit, JHEP 02 (2013) 127 [arXiv:1210.1110] [INSPIRE].

[41] C.D. White, Factorization properties of soft graviton amplitudes, JHEP 05 (2011) 060 [arXiv:1103.2981] [INSPIRE].

[42] S. Melville, S.G. Naculich, H.J. Schnitzer and C.D. White, Wilson line approach to gravity in the high energy limit, Phys. Rev. D 89 (2014) 025009 [arXiv: 1306.6019] [InSPIRE].

[43] A. Luna, S. Melville, S.G. Naculich and C.D. White, Next-to-soft corrections to high energy scattering in QCD and gravity, JHEP 01 (2017) 052 [arXiv: 1611.02172] [INSPIRE].

[44] R. Saotome and R. Akhoury, Relationship between gravity and gauge scattering in the high energy limit, JHEP 01 (2013) 123 [arXiv:1210.8111] [INSPIRE].

[45] A. Sabio Vera, E. Serna Campillo and M.A. Vazquez-Mozo, Color-kinematics duality and the Regge limit of inelastic amplitudes, JHEP 04 (2013) 086 [arXiv:1212.5103] [INSPIRE].

[46] H. Johansson, A. Sabio Vera, E. Serna Campillo and M.A. Vázquez-Mozo, Color-kinematics duality in multi-Regge kinematics and dimensional reduction, JHEP 10 (2013) 215 [arXiv: 1307.3106] [INSPIRE].

[47] H. Johansson, A. Sabio Vera, E. Serna Campillo and M.A. Vazquez-Mozo, Color-kinematics duality and dimensional reduction for graviton emission in Regge limit, in International Workshop on Low X Physics (Israel 2013), Eilat, Israel, 30 May-4 June 2013 [arXiv:1310.1680] [INSPIRE]. 
[48] Z. Bern and A.K. Grant, Perturbative gravity from QCD amplitudes, Phys. Lett. B 457 (1999) 23 [hep-th/9904026] [INSPIRE].

[49] C. Cheung and G.N. Remmen, Twofold symmetries of the pure gravity action, JHEP 01 (2017) 104 [arXiv: 1612.03927] [INSPIRE].

[50] C. Cheung and G.N. Remmen, Hidden simplicity of the gravity action, JHEP 09 (2017) 002 [arXiv: 1705.00626] [INSPIRE].

[51] C. Cheung, C.-H. Shen and C. Wen, Unifying relations for scattering amplitudes, JHEP 02 (2018) 095 [arXiv: 1705.03025] [INSPIRE].

[52] R. Monteiro, D. O'Connell and C.D. White, Black holes and the double copy, JHEP 12 (2014) 056 [arXiv: 1410.0239] [INSPIRE].

[53] A. Luna, R. Monteiro, D. O'Connell and C.D. White, The classical double copy for Taub-NUT spacetime, Phys. Lett. B 750 (2015) 272 [arXiv:1507.01869] [INSPIRE].

[54] A. Luna, R. Monteiro, I. Nicholson, D. O'Connell and C.D. White, The double copy: Bremsstrahlung and accelerating black holes, JHEP 06 (2016) 023 [arXiv:1603.05737] [INSPIRE].

[55] A. Luna, R. Monteiro, I. Nicholson and D. O'Connell, Type D spacetimes and the Weyl double copy, Class. Quant. Grav. 36 (2019) 065003 [arXiv: 1810.08183] [INSPIRE].

[56] A.K. Ridgway and M.B. Wise, Static spherically symmetric Kerr-Schild metrics and implications for the classical double copy, Phys. Rev. D 94 (2016) 044023 [arXiv: 1512.02243] [INSPIRE].

[57] D. Neill and I.Z. Rothstein, Classical space-times from the S matrix, Nucl. Phys. B 877 (2013) 177 [arXiv: 1304.7263] [INSPIRE].

[58] A. Luna et al., Perturbative spacetimes from Yang-Mills theory, JHEP 04 (2017) 069 [arXiv: 1611.07508] [INSPIRE].

[59] A. Luna, I. Nicholson, D. O'Connell and C.D. White, Inelastic black hole scattering from charged scalar amplitudes, JHEP 03 (2018) 044 [arXiv:1711.03901] [INSPIRE].

[60] W.D. Goldberger and A.K. Ridgway, Radiation and the classical double copy for color charges, Phys. Rev. D 95 (2017) 125010 [arXiv:1611.03493] [InSPIRE].

[61] W.D. Goldberger, S.G. Prabhu and J.O. Thompson, Classical gluon and graviton radiation from the bi-adjoint scalar double copy, Phys. Rev. D 96 (2017) 065009 [arXiv:1705.09263] [INSPIRE].

[62] W.D. Goldberger and A.K. Ridgway, Bound states and the classical double copy, Phys. Rev. D 97 (2018) 085019 [arXiv:1711.09493] [INSPIRE].

[63] W.D. Goldberger, J. Li and S.G. Prabhu, Spinning particles, axion radiation and the classical double copy, Phys. Rev. D 97 (2018) 105018 [arXiv:1712.09250] [INSPIRE].

[64] Z. Bern, C. Cheung, R. Roiban, C.-H. Shen, M.P. Solon and M. Zeng, Scattering amplitudes and the conservative Hamiltonian for binary systems at third post-Minkowskian order, Phys. Rev. Lett. 122 (2019) 201603 [arXiv:1901.04424] [inSPIRE].

[65] D. Chester, Radiative double copy for Einstein-Yang-Mills theory, Phys. Rev. D 97 (2018) 084025 [arXiv: 1712.08684] [INSPIRE].

[66] C.-H. Shen, Gravitational radiation from color-kinematics duality, JHEP 11 (2018) 162 [arXiv: 1806.07388] [INSPIRE]. 
[67] J. Plefka, J. Steinhoff and W. Wormsbecher, Effective action of dilaton gravity as the classical double copy of Yang-Mills theory, Phys. Rev. D 99 (2019) 024021 [arXiv: 1807.09859] [INSPIRE].

[68] D.S. Berman, E. Chacón, A. Luna and C.D. White, The self-dual classical double copy and the Eguchi-Hanson instanton, JHEP 01 (2019) 107 [arXiv: 1809.04063] [INSPIRE].

[69] C.D. White, Exact solutions for the biadjoint scalar field, Phys. Lett. B 763 (2016) 365 [arXiv: 1606.04724] [INSPIRE].

[70] P.--J. De Smet and C.D. White, Extended solutions for the biadjoint scalar field, Phys. Lett. B 775 (2017) 163 [arXiv: 1708.01103] [INSPIRE].

[71] N. Bahjat-Abbas, R. Stark-Muchão and C.D. White, Biadjoint wires, Phys. Lett. B 788 (2019) 274 [arXiv: 1810.08118] [INSPIRE].

[72] A. Anastasiou, L. Borsten, M.J. Duff, L.J. Hughes and S. Nagy, Yang-Mills origin of gravitational symmetries, Phys. Rev. Lett. 113 (2014) 231606 [arXiv:1408.4434] [INSPIRE].

[73] L. Borsten and M.J. Duff, Gravity as the square of Yang-Mills?, Phys. Scripta 90 (2015) 108012 [arXiv: 1602.08267] [INSPIRE].

[74] A. Anastasiou et al., Twin supergravities from Yang-Mills theory squared, Phys. Rev. D 96 (2017) 026013 [arXiv:1610.07192] [INSPIRE].

[75] A. Anastasiou, L. Borsten, M.J. Duff, A. Marrani, S. Nagy and M. Zoccali, Are all supergravity theories Yang-Mills squared?, Nucl. Phys. B 934 (2018) 606 [arXiv: 1707.03234] [INSPIRE].

[76] A. Anastasiou, L. Borsten, M.J. Duff, A. Marrani, S. Nagy and M. Zoccali, The mile high magic pyramid, arXiv:1711.08476 [INSPIRE].

[77] A. Anastasiou, L. Borsten, M.J. Duff, S. Nagy and M. Zoccali, Gravity as gauge theory squared: a ghost story, Phys. Rev. Lett. 121 (2018) 211601 [arXiv:1807.02486] [INSPIRE].

[78] S. Nagy, Chiral squaring, JHEP 07 (2016) 142 [arXiv:1412.4750] [INSPIRE].

[79] G.L. Cardoso, S. Nagy and S. Nampuri, A double copy for $N=2$ supergravity: a linearised tale told on-shell, JHEP 10 (2016) 127 [arXiv:1609.05022] [INSPIRE].

[80] G. Lopes Cardoso, G. Inverso, S. Nagy and S. Nampuri, Comments on the double copy construction for gravitational theories, PoS (CORFU2017) 177 (2018) [arXiv: 1803.07670] [INSPIRE].

[81] M. Carrillo-González, R. Penco and M. Trodden, The classical double copy in maximally symmetric spacetimes, JHEP 04 (2018) 028 [arXiv:1711.01296] [INSPIRE].

[82] N. Bahjat-Abbas, A. Luna and C.D. White, The Kerr-Schild double copy in curved spacetime, JHEP 12 (2017) 004 [arXiv:1710.01953] [InSPIRE].

[83] M. Bañados, C. Teitelboim and J. Zanelli, The black hole in three-dimensional space-time, Phys. Rev. Lett. 69 (1992) 1849 [hep-th/9204099] [INSPIRE].

[84] H. Stephani, D. Kramer, M.A. MacCallum, C. Hoenselaers and E. Herlt, Exact solutions of Einstein's field equations, Cambridge University Press, Cambridge, U.K. (2003) [INSPIRE].

[85] S. Giddings, J. Abbott and K. Kuchar, Einstein's theory in a three-dimensional space-time, Gen. Rel. Grav. 16 (1984) 751 [InSPIRE]. 
[86] A.A. García-Díaz, Exact solutions in three-dimensional gravity, Cambridge University Press, Cambridge, U.K. (2017) [INSPIRE].

[87] M. Bañados, M. Henneaux, C. Teitelboim and J. Zanelli, Geometry of the $(2+1)$ black hole, Phys. Rev. D 48 (1993) 1506 [Erratum ibid. D 88 (2013) 069902] [gr-qc/9302012] [INSPIRE].

[88] H. Kim, Spinning BTZ black hole versus Kerr black hole: a closer look, Phys. Rev. D 59 (1999) 064002 [gr-qc/9809047] [INSPIRE].

[89] Z. Bern, J.J. Carrasco, W.-M. Chen, H. Johansson and R. Roiban, Gravity amplitudes as generalized double copies of gauge-theory amplitudes, Phys. Rev. Lett. 118 (2017) 181602 [arXiv: 1701.02519] [INSPIRE].

[90] Y. Verbin, Lower dimensional gravity, Phys. Rev. D 50 (1994) 7318 [INSPIRE].

[91] J.D. Barrow, A.B. Burd and D. Lancaster, Three-dimensional classical spacetimes, Class. Quant. Grav. 3 (1986) 551.

[92] J.D. Bekenstein, Transcendence of the law of baryon-number conservation in black hole physics, Phys. Rev. Lett. 28 (1972) 452 [INSPIRE].

[93] S.W. Hawking, Black holes in the Brans-Dicke theory of gravitation, Commun. Math. Phys. 25 (1972) 167 [INSPIRE].

[94] J.D. Bekenstein, Novel "no-scalar-hair" theorem for black holes, Phys. Rev. D 51 (1995) R6608 [INSPIRE].

[95] G.W. Gibbons and D.A. Rasheed, Dyson pairs and zero mass black holes, Nucl. Phys. B 476 (1996) 515 [hep-th/9604177] [INSPIRE].

[96] M.E. Rodrigues and Z.A.A. Oporto, Thermodynamics of phantom black holes in Einstein-Maxwell-dilaton theory, Phys. Rev. D 85 (2012) 104022 [arXiv:1201.5337] [INSPIRE]. 